\title{
Heat flux effect on photoenhanced and dark silver motion in a thin $\mathrm{As}_{2} \mathrm{~S}_{3}$ layer
}

\author{
A.A. Kudryavtsev, E.V. Michailovskaya \\ Institute of Semiconductor Physics, NAS, Ukraine, \\ Prospekt Nauki 45, Kyiv-28, 03028, Ukraine
}

\begin{abstract}
The influence of a heat flux on the rate of photoenhanced and dark Ag transfer in the lightsensitive thin layer $\mathrm{As}_{2} \mathrm{~S}_{3}-\mathrm{Ag}$ structure was investigated. It was found that the Ag flux increased when a heat flux had the same direction and fell down when the heat flux had an opposite direction. The changes of Ag flux were proportional to a power of the heat flux caused by some temperature difference on the structure surfaces. Coupling between Ag and heat fluxes was described phenomenologically using a generalized mass transfer equation, but the obtained results did not enable to deduce an explicit micromechanism of such interaction. Tentatively, a phonon drag mechanism seems to be more preferable among the known ones, when explaining the Soret effect in this structure.
\end{abstract}

Keywords: mass transfer, Soret effect, photoenhanced diffusion, dark diffusion, chalcogenides, mobile silver ions.

Paper received 04.11.00; revised manuscript received 07.12.00; accepted for publication 12.12.00.

\section{Introduction}

It is known that photodoping, i.e. photoenhanced transfer of metal from the surface into the bulk, takes place in the thin layer chalcogenide vitreous semiconductor (ChVS)-Ag structure [1]. The photoelectric model of this phenomenon based on the latest experimental data was suggested [2]. The model was formulated for the case of uniform temperature field along the metal transfer coordinate. But as it follows from the generalized Wagner equation [3], ions transfer in the bulk of solids is determined by not only electrochemical field but by the temperature field, too. A non-uniform distribution of temperature along the transfer coordinate may arise under usual photodoping due to a non-uniform distribution of light energy absorbed. A corresponding heat flow is able to influence mass transfer. This phenomenon was found by us in the $\mathrm{As}_{2} \mathrm{~S}_{3}-\mathrm{Ag}$ structure [4], and it was qualitatively shown that the passing heat flow accelerates $\mathrm{Ag}$ transfer into the semiconductor bulk by several times, and the reverse heat flow slows it down. The main purpose of this work was to investigate characteristics of the photostimulated and dark Ag-flows in the presence of the heat fluxes in this $\mathrm{As}_{2} \mathrm{~S}_{3}-\mathrm{Ag}$ structure with the aim to get more information upon this effect.

It is worth to emphasize that up to date we know very little about micromechanisms of phonon flux effects on mass transfer in solids. Despite the fact that the Soret effect is well known for a long time, its microscopical nature remains unclear. Renamed can be several possible mechanisms capable to explain observed relations between matter and phonon fluxes, namely: $i$ ) the WirtzHiby mechanism [5] and ii) the phonon-drag one [6]. Both these mechanisms explain direct drag of atomic particles in solids. Besides, there are two opportunities more: iii) transport of ions in the thermoelectric field arising as a result of phonon drag applied to mobile electronic carriers, i.e., as a consequence of the Gurevich effect [7], and iv) transfer of momentum from the electronic to ionic subsystem in the result of the so-called electronic Soret effect, i.e. in the course of electronic carrier scattering on mobile ions [6]. These are indirect mechanisms of heat stimulated matter transfer. To verify what mechanism is more valid in explaining heat flow influence on the $\mathrm{Ag}$ transfer in $\mathrm{As}_{2} \mathrm{~S}_{3}$, we need additional quantitative data.

\section{Experimental procedures}

Lightsensitive $\mathrm{As}_{2} \mathrm{~S}_{3}$ structures served as objects of investigation. They consisted of $\mathrm{Ag}$ and $\mathrm{As}_{2} \mathrm{~S}_{3}$ layers deposited by thermal evaporation in vacuum $10^{-3} \mathrm{~Pa}$ with thicknesses $15 \ldots 30$ and $200 \ldots 500 \mathrm{~nm}$, respectively. The layer thickness was controlled during evaporation using a quartz thickness meter with accuracy $\pm 10 \%$. Tempera- 


\section{A.A. Kudryavtsev, E.V. Michailovskaya: Heat flux effect on photoenhanced and ...}

ture difference near $1 \mathrm{~K}$ on external surfaces of such a structure was reached in three different ways. First, absorption of the active light in the metal layer used for heating the structure surface from the Ag-layer side. In this case the temperature of opposite structure surface was kept close to room temperature due to the relatively great mass and sufficient thermal conductivity of a substrate that was in contact with semiconductor layer. When the Ag-layer was the first to be deposited on a substrate, the polished copper plate pressed to an external surface of the semiconductor served as a heat sink. Secondly, the special heat releasing element (HRE) made from the thin layer of Bi deposited by thermal evaporation was used for heating the surface. The Joule heat was caused by passing electric current. Thirdly, the Ag-layer itself served as HRE heated by an electric current. In separate experiments the difference in temperature on structure external surfaces was reached by using substrates with significantly different thermal conductivities (from silica, glass ceramics, glass, sapphire and metal) under constant conditions of the light excitation and the structure geometry.

The principal method of our investigations was the observation of a metal layer expenditure during photodoping processes or dark duffusion. Reduction of the metal thickness during both of these processes was registered using measurements of the system transmission in the IR spectral range $(\lambda=1.13 \mathrm{~mm})$. Preliminary obtained were special calibration curves that indicated relation between Ag-layer thickness and system transmission changes. Measuring the time interval that is necessary for the system to reach some definite level of transmission we could easily determine a rate of the metal thickness changes. Taking into account the geometry of the system and density of silver we determined respective Ag-expenditure. This value was used as the main parameter for comparison of Ag-fluxes when the direction and value of a heat flux were changed in the structure.

The high-pressure mercury lamp $(250 \mathrm{~W})$ with corresponding color filters was used as a light source of different wavelengths for photodoping and IR-probing. The temperature control of the lightsensitive structure surfaces was made using thin-layer Bi-Sb thermocouples with an accuracy of $\pm 0.3 \mathrm{~K}$. Typical schemes of the investigated structures are shown in Fig.1.

\section{Results}

The influence of a heat flow on the Ag transfer into $\mathrm{As}_{2} \mathrm{~S}_{3}$ bulk was found first in the experiments devoted to the photodoping at room temperature. A comparison of Agflow at the metal-semiconductor interface in two possible ways of mutual disposing the structure layers on the substrate and thermostat relatively an Ag-layer heated (Fig.1a). Determining the heat flow direction in the structure, it was accounted that the light absorption was significantly non-uniform and led to the increase of the Aglayer temperature up to $1 \mathrm{~K}$ for the light power density close to $20 \mathrm{~mW} \mathrm{~cm}^{-2}$. It is conditioned by the high value

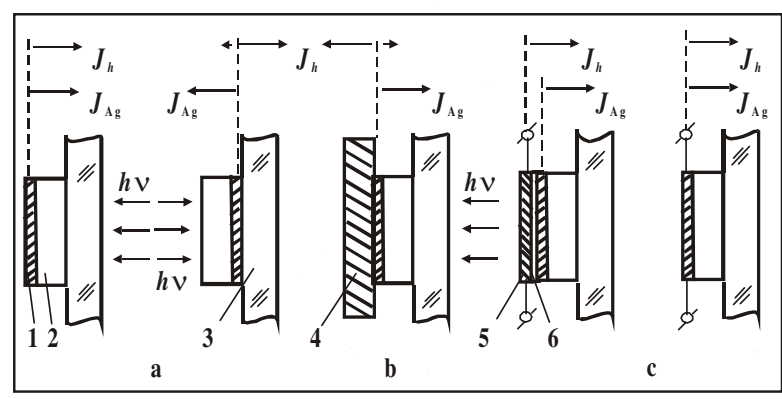

Fig. 1. Types of structures investigated: a - two kinds of mutual disposition for metal and ChVS layers; b - the structure with additive heat sink; $\mathrm{c}-$ structures with heat releasing elements; 1 - Ag-layer; 2 - $\mathrm{As}_{2} \mathrm{~S}_{3}$-layer; 3 - substrate; 4 - copper plate; 5 heat releasing element; 6 - insulating layer. $J_{h}$ and $J_{A g}$ mark the fluxes of heat and silver, respectively.

of the metal absorption coefficient $\left(\sim 10^{6} \mathrm{~cm}^{-1}\right)$ in all considered spectral region. It follows that the main heat release takes place in the thin Ag-layer on the ChVS surface. In the case when metal is disposed from the side of the structure free surface, and the ChVS contacts with the substrate, there are three possible channels of heat exchange: 1) heat transfer to an ambient atmosphere; 2) heat spread along the thin Ag-layer and 3) heat transfer through the ChVS layer to the substrate playing the role of a thermostat. Numeric estimations showed that for the specific geometry of the samples, the heat resistance of two first channels exceeds, by far, the resistance of the third one because of the low thermal conductivity for gas and the small cross-section of the metal layer. Therefore, the predominant heat flow at the metal-ChVS interface ( $\sim 99 \%$ ) is predicted to run through the ChVS-layer to the substrate. In the presence of an additive heat sink (Fig.1b) the main heat flow has an opposite direction.

The comparison of Ag-fluxes on the main stage of the photodoping (when the rate of an Ag-flow in the structure has its maximum) for these two cases with an Ag-flux in the structure having the reverse order of layers contacting with substrate), when heat spreads out of the metal layer in both directions simultaneously, let to make an assumption of the heat flux influence on the Ag transfer. It may be seen in Fig. 2 that the Ag-flux increases when the heat flux has the same direction and falls down when this flux has an opposite direction.

For the further study of a heat flux influence on the Ag transfer, it was necessary to check up the link of the observed Ag-flux changes with the value of the heat flux which, as it follows from the heat conduction theory, is proportional to a temperature gradient under a constant system geometry. From the solution of the heat conduction equation for the structures like shown in Fig.1, it follows that the value of the temperature gradient increases with increasing thermal conductivity of the substrate material. Therefore, samples of the same structure were deposited simultaneously on substrates with significantly different thermal conductivities. Under con- 


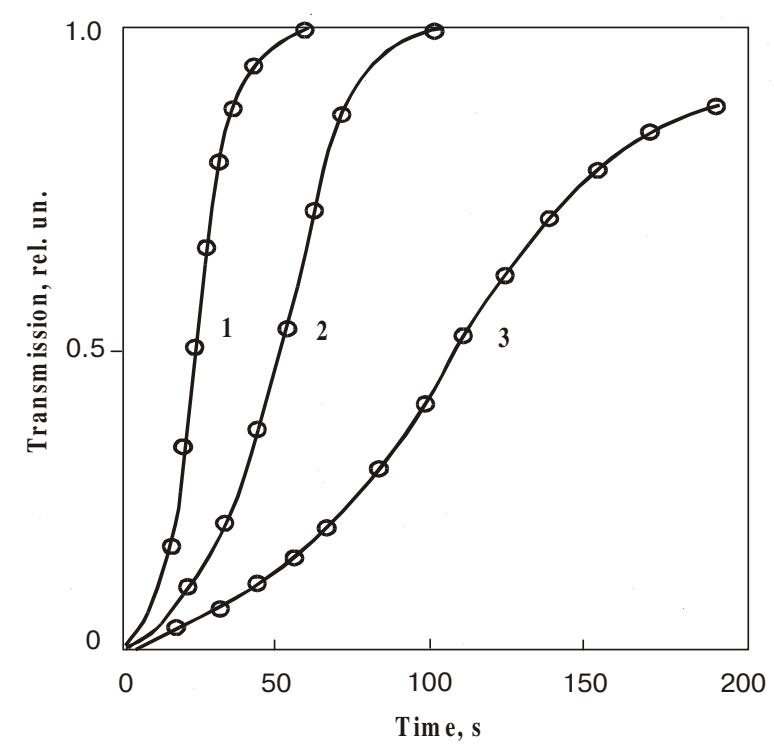

Fig. 2. Kinetic dependences of structure transmission $(\lambda=1.13$ $\mu \mathrm{m})$ during photodoping: $1-$ in the presence of the passing heat flow; 2 - without any appreciable heat flow; 3 - with the reverse heat flow. Lines are drawn as a guide to the eye.

ditions when the heat flux towards the substrate coincided in direction with the photoenhanced Ag-flux, the value of the latter one increased monotonically with a thermal conductivity increase, i.e., with the average value of the temperature gradient along the transfer coordinate (Fig. 3). This result suggests that the Ag-flow should increase with increase of the passing heat flow.

The difficulty with the experiments described above was that they enabled us to find only qualitative link of the Ag transfer rate with the change of the direction and the value of a heat flow in the structure. This difficulty was caused by the fact that the incident light is a heat source and a mass transfer photoenhancer at the same time. Thus, the heat and Ag flows were caused by the same factor, i.e. by power of an incident light. To describe the link between them, it is necessary to control heat fluxes independently. With this aim, structures including HRE (Fig. 1c) were prepared. Using them, the dependence of the Ag-flux values under constant conditions of light excitation on the heat releasing power that determined heat flux magnitudes was obtained. As seen in Fig. 4, this dependence is linear in the range of the heat fluxes up to $1 \mathrm{~W} \mathrm{~cm}^{-2}$. This plot was obtained using the structure shown in Fig. 1c (on the left). But the identical behavior was obtained with the structure represented on the right hand of the same figure. So far as the temperature gradient along $\mathrm{Ag}$ transfer coordinate is proportional to a heat releasing power value, the character of obtained dependence corresponds to the main transfer equation [3].

Additional experimental data upon acceleration of the mass transfer rate were obtained using the structures where the silver layer itself served as HRE. Beginning from the power of a heat flow closed to $100 \mathrm{~mW} \mathrm{~cm}^{-2}$, it was possible to observe silver flux values in the range of

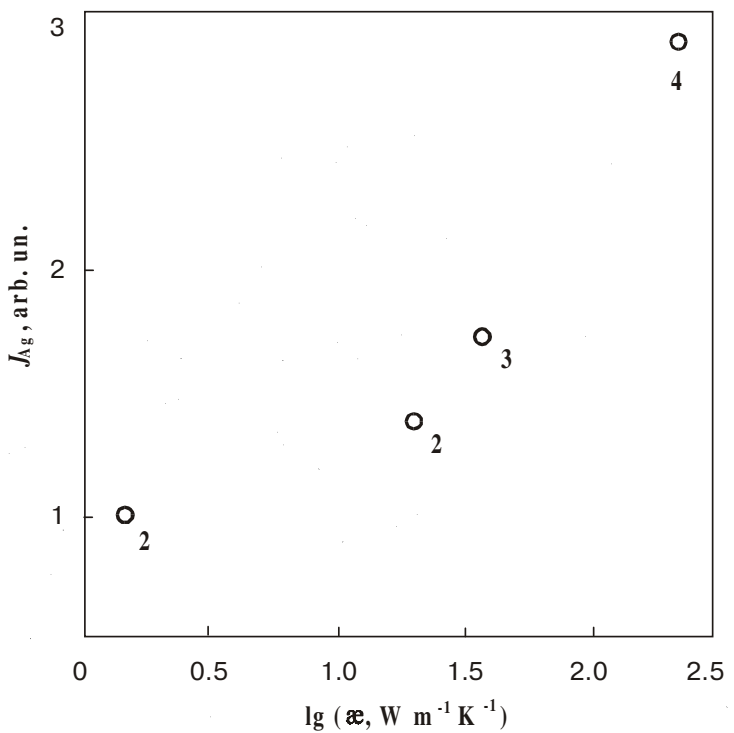

Fig. 3. Magnitudes of Ag-fluxes versus thermal conductivities of specific substrates: 1 - silica; 2 - thick Ni-film; 3 - sapphire plate; 4 - thick Ag-film.

$10^{14}$ to $10^{15} \mathrm{~cm}^{-2} \mathrm{~s}^{-1}$ even in the absence of any light excitation. As shown in Fig. 5, the kinetic dependence of the silver expense under heat stimulation has only the character of a monotonically growing function. At the initial point, immediately after switching on the HRE, the silver flux is maximum, $J_{A g} \sim 10^{15} \mathrm{~cm}^{-2} \mathrm{~s}^{-1}$, but with time it gradually decreases approximately by an order of magnitude, which is associated with increasing the silver concentration in the doped layer above 32 at.\%.

The initial value of the silver flux can be recovered by a short-time illumination of the structure with visible light

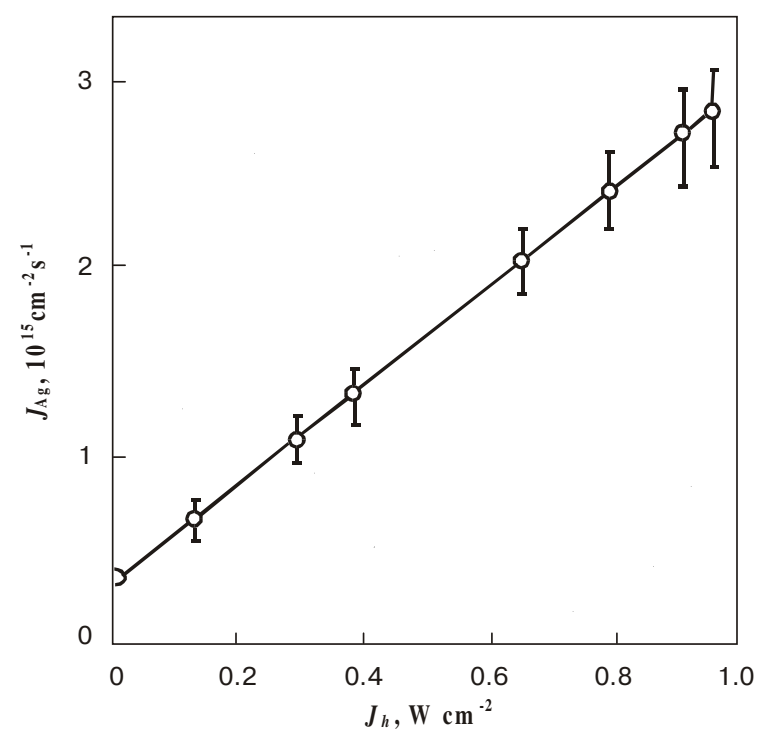

Fig. 4. Dependence between Ag and heat fluxes. The line is drawn as a guide to the eye. 


\section{A.A. Kudryavtsev, E.V. Michailovskaya: Heat flux effect on photoenhanced and ...}

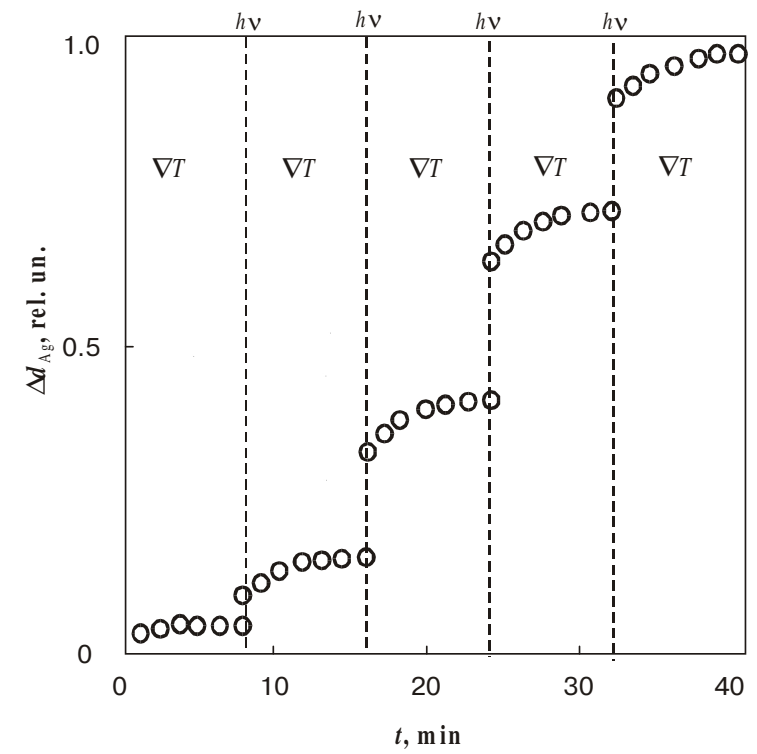

Fig. 5. Dependences of silver expenditure, $\Delta d_{A g}$, on the time of heat flow $(\nabla T)$ action and on short-time exposures by active light $(h v)$.

having the power density close to $20 \mathrm{~mW} \mathrm{~cm}^{-2}$, after that the kinetics had the previous shape again. Alternating these influences, one can exhaust the silver layer completely.

At an increasing power of the heat flow above $500 \mathrm{~mW} \mathrm{~cm}{ }^{-2}$, the initial rate of the silver expenditure changed less during doping, and silver was quickly exhausted without any illumination. This result suggests that even without light excitation, under $1 \mathrm{~K}$ temperature difference, the heat flow should be able to accelerate the silver flow rather significantly (by $10^{5}$ times) as compared with that of room temperature $\left(J_{A g} \sim 10^{10} \mathrm{~cm}^{-2} \mathrm{~s}^{-1}\right)$.

\section{Discussion}

The observed influence of the heat flow on the above mentioned $\mathrm{Ag}$ transfer may be described by the generalized Wagner equation [3] that can be written for the case as

$J_{A g+}=-D_{i} N_{i}\left[\left(\nabla \mu_{i}-q_{i} \nabla \varphi_{d}\right)+q_{i} \nabla \varphi_{l}+U^{*} \nabla T / T\right] / k T$

where $J_{A g+}$ marks the one-dimensional silver ion flux in the given section along the direction which is perpendicular to the layer surface; $D_{i}$ is the coefficient of a joint diffusion for silver ions and electronic carriers; $N_{i}$ and $q_{i}$ are the concentration and charge of mobile ions; $k$ is the Boltzmann constant; $T$ and $\nabla T$ are the symbols of a temperature and its gradient along the transport direction; $\nabla \mu_{i}$ means the gradient of the chemical potential for silver ions; $U^{*}$ is the transfer heat for the same ions; $\nabla \varphi_{d}$ and $\nabla \varphi_{l}$ correspond to dark and light values of electrical potential gradients at the given sections of the structure.

In Eq.(1) the first and second terms outlined by round brackets describe a diffusion-drift equilibrium of silver ions in regions of a sharp change in their concentrations near the interfaces of the metal-doped (M-DS) and dopedundoped (DS-US) semiconductors, while the third and fourth ones determine silver fluxes caused by external light and heat factors, respectively. The most general transfer equation has usually one more term describing a baro-diffusional component, but it was neglected here because of relatively small values of both baro-diffusional coefficient and the pressure gradient $\nabla P$ (in other words, the gradient of mechanical strains in the structure layers have enough time for relaxation during preparation and cannot reach any detectable levels in the course of experimentation because of only $1 \mathrm{~K}$ temperature difference between its surfaces took place).

Eq.(1) permits estimation of Ag-fluxes a priori for different transfer modes and stages of the initial silver layer expenditure if the necessary electrophysical and diffusional characteristics for the structure are known. It is important to note that as-prepared $\mathrm{Ag}-\mathrm{As}_{2} \mathrm{~S}_{3}$ structure is usually characterized by the presence of a very thin intermediate layer between the metal and the undoped semiconductor. This layer arises during the preparation procedure. It leads to the formation of a charged double electric layer near the M-DS interface parameters of which correspond to the known metal - solid electrolyte interface [8].

In the dark at room temperature, in the isotermal conditions, we could observe only a weak diffusional $\mathrm{Ag}$ flow. Its magnitude $\left(J_{A g} \sim 10^{10} \mathrm{~cm}^{-2} \mathrm{~s}^{-1}\right)$ was determined by the disturbance of the diffusion-drift equilibrium for silver ions because of discharging the double layer field by intrinsic electronic carriers. As their concentration was small, the dark diffusional flux of ions was small, too.

The same conclusion is valid for the stage when the doped layer (of the $\mathrm{As}_{2} \mathrm{~S}_{3} \mathrm{Ag}_{2.4}$ composition) occupies already a significant part of the semiconductor bulk. In the dark, in the absence of any temperature difference, the silver flux observed in experiments was small too. The most strong «resistance» to the transfer is created now by the interface between doped and undoped areas where the diffusion - drift equilibrium of ions takes place in the Nernst field caused by higher ionic mobility as compared to the electronic one for $\mathrm{As}_{2} \mathrm{~S}_{3}$ [9]. A small concentration of the intrinsic electronic carriers partly compensates this field and causes a weak ion flux in the field of an electrochemical potential.

A small temperature increase in the metal layer leads to significant rising of the temperature gradient and an appearance of some thermodiffusional component in the mass flux that is described by the fourth term in Eq. (1). Having taken into account the approximate equality between the ion transfer heat and the activation energy for ion conductivity values (from 0.3 to $0.8 \mathrm{eV}$ ) under the condition of the persistency of the diffusion-drift equilibrium near the DS - US interface (when the terms in the round brackets are mutually compensated), we obtained the value $J_{A g} \sim 10^{15} \mathrm{~cm}^{-2} \mathrm{~s}^{-1}$ for thermodiffusional silver flux at $\nabla T \approx 10^{5} \mathrm{~K} \mathrm{~cm}^{-1}$, which was consistent with experimental data. 


\section{A.A. Kudryavtsev, E.V. Michailovskaya: Heat flux effect on photoenhanced and ...}

The role of structure illumination in isothermal conditions can be reduced to generation of the additional electric field in the region of the heterojunction at the DS US interface [2]. This field has a direction and magnitude $\left(\sim 10^{4} \mathrm{~V} \mathrm{~cm}^{-1}\right)$ which provides the accelerated ion transfer into the undoped part of the semiconductor. The corresponding additional flow is caused by the third term in the Eq. (1). The estimation of its value using the data about photo-e.m.f. (10 to $100 \mathrm{mV}$ )[8], the heterojunction thickness $(\sim 60 \mathrm{~nm})[2]$, and the value of the $\mathrm{Ag}^{+}$-ion effective charge, $q_{i}=(0.7$ to 0.8$)|e|$, where $e$ means the electron charge [8], leads to $J_{A g} \sim 10^{15} \mathrm{~cm}^{-2} \mathrm{~s}^{-1}$, which is also consistent with the obtained experimental data.

Under the simultaneous influence of light and temperature fields the silver flux, as compared with the pure photoenhanced one, increased by 2 to 5 times and was dependent on the spectral composition of the incident light and power of the heat flux. As the experiment shows (Figs 3 and 4), the additivity of both factors contributions takes place, which is in a good correspondence with the form of Eq.(1).

For further analysis of any micromechanisms responsible for the thermodiffusional silver transport, let us consider mechanisms listed in the Introduction. In our experiments there are no necessary conditions for realization of the Wirtz-Hiby mechanism as far as it is based on the difference of the chemical potential values in «hot» and «cold» regions of the structure, which might be conditioned by any significant temperature difference. In our case, this difference is approximately $1 \mathrm{~K}$, which cannot provide necessary difference of the chemical potentials.

The direct ion-phonon drag can take place due to resonant scattering of phonons on impurity atoms at coincidence of the matrix atom vibration frequency and a local vibration frequency of the impurity atoms [10]. Such a coincidence provides more effective momentum transfer from the phonon subsystem to the ionic one. The proximity of values of the vibration frequencies for $\mathrm{AsS}_{3}$ tetrahedra and $\mathrm{Ag}$ atoms in amorphous $\mathrm{As}_{2} \mathrm{~S}_{3}$ is known from the experiment [11].

Phonon scattering is also favored by a greater mass of impurity atom compared with the matrix ones [10]. This relation is also valid here, as the mass of silver atom is greater than that of the arsenic and sulfur ones.

On the other hand, the possibility to control silver motion in this structure, redistributing the electronic carriers, has already been argued in experiments with the transport of $\mathrm{Ag}^{+}$-ions in the Dember field [12] and confirmed by efficiency of the photoelectric model for photodoping [2]. But up to date no evidences of the Gurevich effect manifestation in chalcogenides exist. That casts doubt on the applicability of relevant mechanism.

Considering the fourth possibility we should take into account that it can be realized only in substances with a high concentration of electronic carriers (like metals). But in $\mathrm{As}_{2} \mathrm{~S}_{3}$ layers doped with silver the concentration of electronic carriers (holes) does not exceed $10^{19} \mathrm{~cm}^{-3}$ which is far lower than that in metals $\left(10^{22} \ldots 10^{23} \mathrm{~cm}^{-3}\right)$. It means that this mechanism can be neglected, too.
It follows from the above consideration that the mechanism of the direct ion-phonon drag seems to be the most probable one.

\section{Conclusions}

Summing up the obtained results we can state that the proposed phenomenological model permits to quantitatively describe mass transfer in the thin-layer $\mathrm{As}_{2} \mathrm{~S}_{3}-\mathrm{Ag}$ structure at presence of a non-uniform temperature field. Deduced estimates for the rate of silver motion coincide rather well with experimental data obtained in regimes of dark and light thermodiffusion. Moreover, this model enables us to explain from the only viewpoint both known earlier and recent experimental data associated with the silver ion transport in this structure. Total combination of obtained results permits also to conclude that the direct ion-phonon drag is a most probable micromechanism of the Soret effect in the studied structure.

\section{Acknowledgments}

Authors express their sincere gratitude to Dr. T.A. Kudykina and Prof. I.Z. Indutnyi for fruitful discussions of obtained results.

\section{References}

1. M.T. Kostishin, E.V.Michailovskaya and P.F. Romanenko, Effect of photographic sensitivity in thin semiconductor layers deposited on metallic substrates // Soviet Physics: Solid State 8(2), pp. 571-572 (1966).

2. I.Z. Indutnyi, V.A. Dan'ko, A.A. Kudryavtsev, E.V. Michailovskaya and V.I. Min'ko, Photodoping in $\mathrm{As}_{2} \mathrm{~S}_{3}-\mathrm{Ag}$ structures // J.Non-Cryst. Solids 185(2), pp. 176 - 182 (1995).

3. V.N. Chebotin, M.V. Perfil'ev, Electrochemistry of solid electrolytes, Chemistry, Moscow, 1978 (in Russian).

4. A.A. Kudryavtsev, E.V. Michailovskaya, Silver photodiffusion in non-homogeneous temperature field of the thin-layer ChVS-Ag structure. In: Abstracts of the International SchoolConference on Physical Problems in Materials Science of Semiconductors "PPMSS'95», Chernivtsi, Ukraine, 11-16 September 1995, p.244.

5. K.Wirtz, J.W. Hiby, Phys. Zs. 44 (221), 369 (1943).

6. V.B. Fiks, Ionic conductivity in metals and semiconductors, Nauka, Moscow, 1969 (in Russian).

7. A.I. Anselm, Introduction to the theory of semiconductors, GIFML, Moscow, 1962 (in Russian).

8. V.A. Dan'ko, I.Z. Indutnyi, O.P. Kasyarum, V.V. Kolomiets, A.A.Kudryavtsev and V.I. Min'ko, Photostimulated processes in lightsensitive systems ChVS - Ag, Preprint No 8-89, Institute of Semiconductors, NAS of Ukraine, Kiev, 1989, 26p. (in Russian).

9. H. Kahnt, F. Schirmeister and A. Feltz, Model of photostimulated diffusion of $\mathrm{Ag}$ in amorphous chalcogenides // Phys. Stat. Solidi (a) 108 (2), pp. 149 - 153 (1988).

10. V.S. Oskotsky and I.A.Smirnov, Defects in crystals and thermoconductivity, Nauka, Leningrad, 1972 (in Russian).

11. A.I.Stetsun, I.Z.Indutnyi and V.G.Kravets, Infrared absorption of Ag- and Cu-photodoped chalcogenide films // J.NonCryst. Solids 202(2), pp. 113 - 121 (1996).

12. O.P. Kasyarum and A.A. Kudryavtsev, Mechanism of photostimulated Ag-migration in a bulk of ChVS. In: Proc. Int. Conf. «Non-crystalline semiconductors -89 », Vol. 2, PIK «Patent», Uzhgorod, 1989, p.227. 